\title{
FiRST REPORTS OF THE PSYCHOLOGICAL CONSEQUENCES OF the SARS-CoV-2 Pandemic. What appeared IN THE SCIENTIFIC LITERATURE BETWEEN JANUARY AND JUNE 2020?
}

\author{
Marta Makara-Studzińska, ${ }^{\mathrm{A}, \mathrm{F}}$, Maciej Załuski, ${ }^{\mathrm{B}, \mathrm{D}, \mathrm{D}}$, Katarzyna Adamczyk ${ }^{\mathrm{D}}$, Jakub Lickiewicz ${ }^{\mathrm{B}, \mathrm{C}}$
}

Department of Health Psychology, Institute of Nursing and Midwifery, Jagiellonian University Medical College, Cracow, Poland

Authors' contribution:

A. Study design/planning $\bullet$ B. Data collection/entry $\bullet$ C. Data analysis/statistics $\bullet$ D. Data interpretation $\bullet$ E. Preparation of manuscript $\bullet$ F. Literature analysis/search $\bullet$ G. Funds collection

\author{
Address for correspondence: \\ Dr Maciej Załuski \\ Department of Health Psychology \\ Institute of Nursing and Midwifery \\ Jagiellonian University Medical College \\ Cracow, Poland \\ e-mail: maciej.zaluski@uj.edu.pl \\ SUBMITTED: 16.07 .2021 \\ ACCEPTED: 23.08.2021 \\ DOI: https://doi.org/10.5114/ppiel.2021.110453
}

\begin{abstract}
The SARS-CoV-2 pandemic can be perceived from a biopsychosocial perspective. The biological aspect focuses on groups particularly susceptible to infection, like the elderly, patients treated for severe somatic complaints, and carriers of the SARS-CoV-2 virus. The psychological aspect relates to the negative impact of social isolation and of the information chaos associated with the pandemic. The group of people at risk of developing psychological problems consists of psychiatric patients, persons in quarantine, relatives of patients hospitalized due to COVID-19, carriers of the SARS-CoV-2 virus, and socially lonely people. Hence, many people may experience episodic or chronic emotional disturbances. The social aspect draws attention to the negative psychological consequences of the gradually deteriorating economic situation of citizens and the cumulative multiple consequences of social isolation. The problem undertaken by the authors of the article was to recognize the psychological consequences of the first weeks of the SARS-CoV-2 pandemic in the human population. The article is of an overview nature. The literature used in the article came from the database MEDLINE between 1 January and 30 June 2020. The articles were obtained using the key words: SARS-CoV-2, mental health, and pandemics. The method of narrative analysis was used. This study combines the above-mentioned 3 aspects, showing the presumed long-term psychological consequences of a pandemic for the functioning of the individuals and society. The most important psychological consequences of a pandemic are explained, including the issue of high-risk groups. Probable risk factors for emotional disorders and psychological problems are described.
\end{abstract}

Key words: SARS-CoV-2, mental health, pandemics, public health.

\section{INTRODUCTION}

The beginning of the SARS-CoV-2 epidemic posed a serious challenge for the health system all over the world. The pathogen, belonging to the coronavirus group of RNA viruses, was first identified in 2019 in the Chinese province of Wuhan. Various courses of this illness are possible, ranging from asymptomatic to very serious disease with acute or chronic respiratory failure among patients who were previously diagnosed with chronic obstructive pulmonary disease, coronary artery disease, and/or hypertension [1, 2].

Epidemiologists and medical leaders mobilized all over the world, recommending isolation to limit the spread of the virus, but were soon faced with serious opposition that was primarily linked with allegations that such practices restricted citizens' individual freedoms [3]. Experience gained from previous epidemics, like severe acute respiratory syndrome (SARS) in 2002-2004 or respiratory syndrome (middle east respiratory syndrome - MERS) in the Arabian Peninsula in 2012 and South Korea in 2015, demonstrates a risk for developing mental disorders, signs of stressful suicidal behaviour, as well as domestic and psychoactive substance abuse [4-6].

Have the aforementioned assumptions been confirmed? This article attempts to answer this question. The aim was to recognize the psychological consequences of the SARS-CoV-2 pandemic in the first period of its occurrence. Based on knowledge from scientific articles published in the period: 1 January - 30 June 2020, the relationships between the pan- 
demic and the occurrence of mental dysfunctions, both identification and risk factors, were described in various social groups. The literature used in the article was obtained from the MEDLINE database using the PubMed search engine. The articles were obtained using the following key words: SARS-CoV-2, mental health, and pandemics. The method of narrative meta-analysis of articles was used. These types of reviews, as opposed to a systematic review, are used to obtain a broad view on a topic, less in finishing quantitative answers to specific clinical questions [7]; they were used when studies were scant or preliminary. The selection process for the narrative literature reviewed is not usually strictly specified, the evaluation process is variable not rigorously critical, and the synthesis is qualitative not quantitative, and therefore it is subject to systematic and random error.

Since the declaration of a pandemic, research began in many countries around the world to assess its association with the mental health of citizens. The first analyses carried out among the inhabitants of China, Iran, Saudi Arabia, Spain, Italy, and Brazil, as well as other countries of the world, showed that the risk of the SARS virus significantly interferes with everyday family, social, and professional functioning, reducing the quality of life [4, 8-13]. The scope and ultimate impact of the pandemic on global human health, the economy, and social cohesion are unknown, although the first scientific reports on these topics are already being prepared $[4,13,14]$. Factors contributing to the development of emotional disorders in people in connection with the pandemic include the unpredictable nature of the spread of the virus and the introduced sanitary restrictions that impede free access to social resources. The fear of infection can vary in severity. Moderate fear activates a person to take actions that reduce the risk of infection, too much fear focuses primarily on reducing it at the expense of taking steps to remove the source, too little fear loses the function of a threat signal and does not strengthen the motivation to act.

The conducted analysis of the literature identified 2 groups of people with an increased risk of developing mental health problems in connection with the state of the pandemic [15]. The first group consists of somatically healthy people, whose emotional problems may result from fear of illness and death, concern for the health and life of relatives, loss of resources, social exclusion, as well as the need to comply with the safety rules introduced during the pandemic. The restrictions concern rules of movement, the obligation to undergo quarantine, restrictions on social life, changes in the organization of work of medical care and rehabilitation units, social welfare and education, restrictions on crossing borders and international traffic, and the organization of commercial and service establishments, sports and recre- ation. The second group consists of people requiring psychiatric treatment and people receiving psychiatric treatment before the outbreak of the pandemic.

\section{HEALTHY PEOPLE LIVING IN THE PANDEMIC AREAS}

A study conducted in China at the turn of January and February 2020 in a group of 52,000 people showed that $35 \%$ of respondents had symptoms of psychological stress, of which $5 \%$ had very high severity [16]. Approximately 5\% (4.6\%) of respondents reported post-traumatic stress disorders (PTSD) in the first month after the outbreak of a pandemic, rising to $7 \%$ in the areas with the greatest spread of the virus $[17,18]$. The analysis of the content of online posts from 10,000 Internet users in China before and after the declaration of a pandemic, conducted in January 2020 , showed an increase in the intensity of negative emotions (anxiety, anger, sadness) and a decrease in the intensity of positive emotions and life satisfaction [19]. The analyses made on a representative Belgian sample aged between 18 and 65 years showed a $10 \%$ increase in the number of respondents with signs of stress after the introduction of mobility restrictions [20]. Reports from epidemiological studies conducted in Italy in April 2020 indicated that 19.4\% of respondents had symptoms of mild psychological stress and $18.6 \%$ had severe symptoms of stress [12]. In Ethiopia, there was a 300\% increase in the incidence of depression symptoms compared to pre-pandemic data [21]. Similarly, in a group of 500 quarantined people in Brazil, increased symptoms of depression, anxiety, and stress were observed [13]. Among 1160 Saudi Arabians, $23.6 \%$ reported moderate to severe PTSD symptoms, $28 \%$ moderate to severe depressive symptoms, $24 \%$ anxiety symptoms, and $22.3 \%$ psychological stress at the beginning of the virus epidemic [9].

It is assumed that the pandemic and the related limitations will intensify the occurrence of mental disorders from the group of neurotic, stress-related, and somatic disorders [22]. The anxiety-generating thoughts concern the possibility of death as a result of COVID-19 disease, the risk of accidental contact with a virus carrier, social isolation orders due to quarantine, depressing social stigmatization of a person-carrier of the SARS-CoV-2 virus, a person treated for COVID-19 or staying in quarantine, lack of food, and job loss or bankruptcy as a result of the restrictions introduced. By analysing people's reactions in the following weeks of the Wuhan pandemic, researchers identified 3 sources of social anxiety [16]. The fear was started by confirming the supposition that the virus had adapted well to infect humans. As a result, among patients with dominance of psychotic defence mechanisms, transient delusional thinking disorders and the presence of obsessive-compulsive 
behaviours were observed. In the following weeks, the introduction of strict quarantine of healthy people due to the possibility of infection and other sanitary restrictions became a source of concern. The entire city was isolated and the possibility of social contact between its inhabitants was highly limited. The announcement on 30 January 2020 of the World Health Organization's declaration on the outbreak of COVID-19 (Public Health Emergency of International Concern - PHEIC) also raised concerns. An increased number of people seeking help for obsessive-compulsive symptoms has been observed in many countries. This applied in particular to residents subject to restrictive sanitary regulations requiring frequent hand washing, disinfection of door handles in public buildings, and even locks on doors and keys [22].

Reports from studies on the prevalence and severity of anxiety disorders in people affected by the COVID-19 pandemic showed a significant increase compared to the pre-pandemic state. In a group of Chinese students, $25 \%$ were diagnosed with anxiety of varying severity [23]. The results of the diagnosis carried out at the turn of January and February 2020 in a group of 1210 Chinese residents indicated the presence of anxiety symptoms in the first weeks of the pandemic in $36 \%$ of respondents, of which $8.4 \%$ were of extremely high intensity [16]. A diagnosis carried out in Iran in March 2020 in a sample of 10,754 people showed that $9.3 \%$ of the respondents represented severe anxiety and $9.8 \%$ had very severe anxiety [11].

\section{HEALTHY PEOPLE IN QUARANTINE AND DIAGNOSED WITH SARS-COV-2 INFECTION}

Quarantine is the compulsory, temporary isolation of healthy people who are suspected to be carriers of the pathogen. It also includes hospital isolation of sick or suspected carriers. Social separation of people treated for an infectious disease coincides with the loss of physical and mental health. Extending quarantine periods exacerbated the symptoms of PTSD and depression $[24,25]$. People in quarantine were diagnosed with sleep disorders, bouts of anger and frustration, and a feeling of boredom caused by the lack of access to social support resources and physical activity. The negative consequences continued after the end of the isolation period and were closely related to the lack of work $[26,27]$. The SARS-CoV-2 pandemic is a form of catastrophic stressor with often fatal health consequences, causing an extreme threat due to the territorially unlimited range of occurrence and ease of spreading [25]. Biological catastrophes cause symptoms of post-traumatic stress in humans, as well as chronic stress with a negative impact on the sense of security, faith in a fair world [28], self-esteem, optimistic attitude towards the future, and self-confidence. Population studies of people affected by Ebola virus showed a higher probability of occurrence of symptoms of post-traumatic stress disorder, obsessive-compulsive disorder, sleep disorder, suicidal thoughts, psychoactive substance abuse, and social anxiety than in the general population [25]. Approximately $30 \%$ (28.9\%) of Canadians isolated due to virus infection were diagnosed with symptoms of PTSD, while $31.2 \%$ were diagnosed with depressive disorders [26]. An additional factor exacerbating the symptoms was the direct knowledge and personal contact with a patient infected with SARS-CoV-2 virus. People in quarantine reported a decrease in the level of trust in people and a sense of belonging to the local community [29]. Sleep disturbances were a significant problem among quarantined patients. Meanwhile, disturbances in the rhythm and quality of sleep comorbid with anxiety and depressive symptoms may lead to the emergence of auto-aggressive behaviours, being one of the risk factors for the occurrence of suicidal thoughts [21].

\section{PEOPLE REQUIRING PSYCHIATRIC TREATMENT AND PEOPLE PREVIOUSLY TREATED PSYCHIATRICALLY BEFORE THE OUTBREAK OF THE PANDEMIC}

Using information gathered during previous epidemics, an inevitable increase in psychiatric diagnoses in the aftermath of the SARS-CoV-2 pandemic was assumed [16, 30]. Scientists believe that psychological and psychiatric problems can worsen and last much longer than the somatic consequences of spreading the virus [29]. The expected increase in mental disorders and diseases, which may also result in an increase in the number of suicides, may occur in the middle phase and after the end of the SARS-CoV-2 epidemic. It can be expected that increased susceptibility to stress, immature defence mechanisms, and the personality structure of patients predisposing to psychotic disorders may contribute to the aggravation of mental disorders and their recurrence. The situation is worsened by the limitations of the patient's direct contact with a doctor or psychotherapist. A special group is made up of people with diagnosed markers of the presence of the SARS-CoV-2 virus and who needed treatment for both mental and infectious disease [31]. It has been observed that the treatment of a psychiatric patient carrying the SARS-CoV-2 virus is the source of several problems, both therapeutic and organizational. It is possible that a patient with a psychotic or serious personality disorder, who carries the virus, may engage in risky actions against the requirements of isolation. Scientists expected harmful interactions between the psychiatric and internal medicine used. A stay in a psychiatric ward may require supplementing the staff with round-the-clock care 
of an anaesthesiologist and organizational changes enabling a psychiatric patient to have direct contact with a specialized intensive care unit; moreover, the creation of small quarantine units in infectious and intensive care units was postulated with the possibility of providing consultative support for psychiatrists. It is anticipated that patients with cognitive impairment may have difficulties in taking hygiene measures and thus preventing infections. It can be more difficult for them to be socially isolated due to limited possibilities of contact with friends and family. Meanwhile, loneliness is a major risk factor for the death of the elderly [2]. Similar problems related to loneliness may concern people with intellectual disabilities as well as their guardians [32]. Many of the caregivers benefited from the care provided by the patient's daily stay in a care centre. During the pandemic, this possibility was very limited. The vision of a viral infection and hospitalization is an additional source of stress for the caregivers resulting from concerns about the patient's state. It was observed that limitation of access to alcohol led to the occurrence of withdrawal symptoms among alcohol addicted patients, with all its health consequences, as well as the occurrence of suicidal behaviour. Canada has seen a $20 \%$ increase in alcohol consumption by people aged $15-49$ years as a secondary effect of the pandemic [21].

\section{RISK FACTORS OF EMOTIONAL DISORDERS AND THEORIES EXPLAINING THEIR SOURCES}

The severity of the symptoms of emotional disorders depends on the patient's sex, age, education, and place of residence. In epidemiological studies conducted in China in the period December 2019 January 2020, women reported a higher level of anxiety, as well as the presence of symptoms carrying a risk of post-traumatic stress disorder [16]. However, at the same time, the highest mortality was diagnosed among men (2.8\%) as compared to women (1.7\%) [33], which makes the group more vulnerable to psychological stress. Chinese people aged between 21 and 40 years are considered to be at risk of mental disorders and alcohol abuse [5]. Similar data were observed in European countries. In Spain, young men (under 18 years old) and women were indicated as the risk group for mental disorders [4]. Reports from research carried out in India indicated that more women $(66 \%)$ in comparison with men $(34 \%)$ declared the presence of signs of psychological stress as a result of the pandemic [34]. In studies in Iran, the level of anxiety was significantly higher among women, people aged 21-40 years and respondents with higher education [11]. Pregnant and puerperal women are particularly vulnerable to stress, which is related to the fear of limited access to medical servic- es, insufficient social support, and virus infection [16]. It is believed that the necessity to maintain social isolation may contribute to an increase in domestic violence. It has been estimated that the introduction of 6-month restrictions on movement and social contact may coexist worldwide with 31 million additional cases of domestic violence [6].

Increased signs of anxiety and behavioural disorders were commonly recognized in children and adolescents. Children more often reported attention deficit disorder and a sense of loneliness. They withdrew from social life more often and exhibited intensified regressive behaviour (including bedwetting) [34]. The results of reports from Italy and Spain confirmed the negative impact of restrictions caused by the pandemic on the emotional state and behaviour of children [34]. World Health Organization experts stated that children and adolescents were more likely to experience domestic violence during a pandemic, especially physically and mentally disabled children living in orphanages, large human clusters, and those working on the street. Thirty-two per cent of young people living in the British Isles reported a negative impact of the pandemic on their mental health and future life. The main sources of anxiety were concerns about the health of their family members, the further fate of their education due to the closure of schools and universities and the economic collapse, the inability to perform the activities available before the outbreak of the pandemic, and the breakdown of close social relationships [34]. The high level of anxiety among people aged 18-30 years was due to their frequent activity on social media, which is the place where information about the pandemic appeared and spread [35]. The term 'infodemic monikers' means the uncontrolled and rapid expansion of inaccurate messages through electronic media, which generate errors in the perception of the described phenomena or false messages. Mistakes are the source of social prejudices, as in the case of the so-called anti-vaccine agents and the reason for difficulties in combating the virus epidemic. Elderly people and those treated for somatic diseases are at higher risk of life-threatening complications from SARS-CoV-2. In the United States $8 / 10$ people who died were 65 or older, while in Germany that age was 70 . Elderly patients with existing diseases (cardiovascular diseases, hypertension, diabetes) are particularly concerned about the risk of contracting the virus and the lack of access to treatment [19, 32, 33, 36].

People with higher education have declared a higher level of anxiety [35]. It is presumed that the reason is the awareness of the extensive consequences of being infected with COVID-19, as well as the occurrence of difficulties in maintaining the current standard of living and extensive knowledge 
in the field of health education. Concerns about contracting the virus in public transport vehicles, being late to work, and losing income resulted in higher levels of stress and anxiety among city dwellers. For all citizens, the risk factors identified in the first months of the pandemic were as follows: limited access to health care facilities, a decline in the efficiency of regional health and epidemiological systems, and the number of preventive and control measures at the disposal of a given region (masks, carrier testing). Inhabitants of cities located in industrialized regions are exposed to contact with a large number of migrant workers living in suburban towns and villages. As a result, the level of anxiety grew, the content of which was fears of contracting the virus from incoming employees [35].

The type of occupation was indicated as another risk factor. Professions in which an employee has direct contact with large groups of people, medical professions, work in educational institutions, as well as commercial, sports, and cultural institutions, are burdened with a higher risk of infection. Employees' fears for their own health and those of their loved ones are a source of anxiety and mood swings. There are known cases of neighbours stigmatizing doctors or nurses as a result of blaming them as virus carriers. There is also a high risk when engaged in social help towards people affected by the situation: both lack of engagement and too much of it create and intensify stress. The more a person is engaged, the more it creates a linear relationship of depression as an effect of experienced failures. In a study of 1250 health care workers in China, $50.4 \%$ were diagnosed with symptoms of depressive disorders, and $44.6 \%$ were clinical with severe anxiety, $34.0 \%$ reported difficulty falling asleep and frequent waking up, and $71.5 \%$ reported symptoms of psychological stress [21]. Schwartz and Pines [37] examined the feelings of military physicians in their work during a pandemic. The terms "I am exhausted, overwhelmed, and restless" and "the current workload is not sustainable" dominated. A review of the literature published between January and March 2020, for research reports on the prevalence of mental disorders symptoms in the group of health care workers, provided alarming results [38]. Medics reported significant psychological stress in the form of chronic psychological stress, and the presence of symptoms of depression and anxiety. From $2.2 \%$ to $14.5 \%$ of the study participants declared a high intensity of these symptoms. The analysis of risk factors showed the role of age, gender, profession, type of medical specialization, and scope of duties that expose people to close contact with a patient infected with the SARS-CoV-2 virus. Forty-seven per cent of Canadian health professionals reported the need for immediate psychological support regarding the consequences of work performed in the first months of the pandemic. Fifty per cent of medical workers in China were diagnosed with symptoms of depression, $45 \%$ anxiety, and $34 \%$ insomnia. In Pakistan, $43 \%$ of medical workers reported moderate and $26 \%$ severe symptoms of psychological stress [34].

Risk factors also include the socioeconomic status of citizens in a pandemic country. People with a lower economic status are characterized by poorer somatic health, are more often chronically ill, and have limited access to medical care [39]. Financial shortages constitute a barrier to access to drugs, and they encourage patients to make arbitrary changes in their intake, which may lead to treatment discontinuation. Poverty may limit healthy behaviours, which are treated as inconveniences and a source of unnecessary psychosocial stress [40]. This concerns the necessity to follow the recommendations of isolation in the form of keeping a safe distance, using masks, washing hands, and undergoing quarantine. As a result, the health effects of an epidemic may vary according to the socio-economic status of the patient. This situation occurred in 2002-2004 in China during the SARS pandemic, during which low socio-economic status coexisted with the more severe negative psychosocial consequences of the pandemic [41].

\section{SUMMARY}

Research conducted in the first months of the SARS-CoV-2 pandemic among people affected clearly showed negative emotional changes as a response to the occurrence of this catastrophic phenomenon. The information presented in this article indicate that the SARS-CoV-2 virus pandemic was assumed to become a source of social health problems. Time has verified these assumptions by revealing the true extent of the negative emotional changes that humanity is currently struggling with. Reports from research are gradually being published, showing that the first social reactions caused by the virus and the introduced limitations have been supplemented with further ones, verifying the initial assumptions.

As the state of pandemic continues, an increase in in-depth analyses as well as new information on long-term and delayed psychological problems can be expected. New threats have also appeared, such as failure to comply with the introduced restrictions. This is an example of a gradual depletion of psychosocial resources, which is manifested in people by increasing anger and aggression. All the signs of negative emotional changes described in this article affect healthcare professionals in a special way. Both when they meet the patient's emotions and their own emotions evoked by risk of infecting him/herself and his/ her family.

The study did not require the approval of the Bioethics Committee. 


\section{Disclosure}

The authors declare no conflict of interest.

\section{References}

1. Huang C, Wang Y, Li X, et al. Clinical features of patients infected with 2019 novel coronavirus in Wuhan, China. Lancet 2020; 395: 497-506.

2. World Health Organization. WHO Director-General's opening remarks at the media briefing on COVID-19 - 5 January 2021. Available at: <https://www.who.int/director-general/ speeches/detail/opening-remarks-for-the-media-briefingon-covid-19-5-january-2021 (accessed 1 February 2021).

3. Armitage R, Nellums LB. COVID-19 and the consequences of isolating the elderly 2020. Lancet Public Health 2020; 5: e256.

4. Rodriguez-Rey R, Garrido-Hernansaiz H, Collado S. Psychological Impact of COVID-19 in Spain: early data report. Psychol Trauma 2020; 12: 550-552.

5. Ahmed MZ, Ahmed O, Aibao Z, et al. Epidemic of COVID-19 in China and associated Psychological Problems. Asian J Psychiatr 2020; 51: 102092.

6. Usher K, Bhullar N, Durkin J, et al. Family violence and COVID-19: Increased vulnerability and reduced options for support. Int J Ment Health Nurs 2020; 29: 549-552.

7. Cook D, Mulrow C, Haynes B. Systematic reviews: synthesis of best evidence for clinical decisions. Ann Intern Med 1997; 126: 376-380.

8. Wu P, Fang Y, Guan Z, et al. The psychological impact of the SARS epidemic on hospital employees in China: exposure, risk perception, and altruistic acceptance of risk. Can J Psychiatry 2009; 54: 302-311.

9. Alkhamees A, Alrashed S, Alzunaydi A, et al. The psychological impact of COVID-19 pandemic on general population of Saudi Arabia. Compr Psychiatry 2020; 102: 152192.

10. Zandifar A, Badrfam R. Iranian mental health during the COVID-19 epidemic. Asian J Psychiatr 2020; 51: 101990.

11. Moghanibashi-Mansourieh A. Assessing the anxiety level of Iranian general population during COVID-19 outbreak. Asian J Psychiatr 2020; 51: 102076.

12. Mocccia L, Janiric D, Pepea M, et al. Affective temperament, attachment style, and the psychological impact of the COVID-19 outbreak: an early report on the Italian general population. Brain Beh 2020; 87: 75-79.

13. Goncalves A, Zuanazzi A, Salvador A, et al. Preliminary findings on the associations between mental health indicators and social isolation during the COVID-19 pandemic. Arch Psychiatr Psychother 2020; 2: 10-19.

14. Fofana NK, Latif F, Bashir MF, et al. Fear and agony of the pandemic leading to stress and mental illness: an emerging crisis in the novel coronavirus (COVID-19) outbreak. Psychiatry Res 2020; 291: 113230.

15. Sathyanath SM, Sathyanath SM. Stigma reduction and provision of mental health services in the public health response to COVID 19. Indian J Common Health 2020; 32: 269-272.

16. Wang C, Pan R, Wan X, et al. Immediate psychological responses and associated factors during the initial stage of the 2019 coronavirus disease (COVID-19) epidemic among the general population in China. Int J Environ Res Public Health 2020; 17: 1729.

17. Sun L, Sun Z, Wu L, et al. Prevalence and risk factors of acute posttraumatic stress symptoms during the COVID-19 outbreak in Wuhan, China. MedRxiv 2020.
18. Liu N, Zhang F, Wei C, et al. Prevalence and predictors of PTSS during COVID-19 outbreak in China hardest-hit areas: gender differences matter. Psychiatry Res 2020; 287: 112921.

19. Li S, Wang Y, Xue J. The impact of COVID-19 epidemic declaration on psychological consequences: a study on active Weibo users. Int J Environ Res Publ Health 2020; 17: E2032.

20. Hoof DEV. Lockdown is the world's biggest psychological experiment - and we will pay the price, 2020. Available at: https://www.weforum.org/agenda/2020/04/this-isthepsychological-side-of-the-covid-19-pandemic-that-wereignoring/ (accessed 1 May 2020).

21. Sher L. COVID-19, anxiety, sleep disturbances and suicide. Sleep Med 2020; 70: 124.

22. Kumar A, Nayar R. COVID 19 and its mental health consequences. J Ment Health 2020; 180: 817-818.

23. Cao W, Fang Z, Hou G. The psychological impact of the COVID-19 epidemic on college students in China. Psychiatry Res 2020; 287: 112934

24. Hossain M, Sultana A, Purohit N. Mental health outcomes of quarantine and isolation for infection prevention: A systematic umbrella review of the global evidence. Epidemiol Health 2020; 42: e2020038.

25. Fiorillo A, Gorwood P. The consequences of the COVID-19 pandemic on mental health and implications for clinical practice. Eur Psychiatry 2020; 63: 1-4.

26. Hawryluck L, Gold WL, Robinson S, et al. SARS control and psychological effects of quarantine, Toronto, Canada. Emerg Infect Dis 2004; 10: 1206-1212.

27. Huremović D. Mental health of quarantine and isolation. In: Huremović D (Ed.). Psychiatry of pandemics. Springer, Cham 2019; 95-118.

28. Skarżyńska K. Po co ludziom sprawiedliwość? Nadawanie sensu zdarzeniom i złudzenie kontroli. Czasopismo Psychologiczne 2009; 15: 255-265.

29. Xiao H, Zhang Y, Kong D, et al. Social capital and sleep quality in individuals who self-isolated for 14 days during the coronavirus disease 2019 (COVID-19) outbreak in January 2020 in China. Med Sci Monit 2020; 26: e923921.

30. Gunnell D, Appleby L, Arensman E, et al. Suicide risk and prevention during the COVID19 pandemic. Lancet Psychiatry 2020; 7: 468-471.

31. Skoneczny T, Lebiecka Z, Kucharska-Mazur J, et al. Problems with treatment of COVID-19 (+) patient with psychiatric disorder - a case report. Arch Psychiatry Psychother 2020; 2: 7-9.

32. Wright A, Salazar A, Mirica M, et al. The invisible epidemic: neglected chronic disease management during COVID-19. J Gen Intern Med 2020; 35: 2816-2817.

33. Gebhard C, Regitz-Zagrosek V, Neuhauser H, et al. Sex differences in clinical phenotype and transitions of care among individuals dying of COVID-19 in Italy. Biol Sex Differ 2020; 11: 1-13.

34. World Health Organization. Policy brief: COVID-19 and the need for action on mental health. Available at: <https:// www.un.org/sites/un2.un.org/files/un_policy_brief-covid_ and_mental_health_final.pdf> (accessed 1 February 2021).

35. Qiu J, Shen B, Zhao M, et al. A nationwide survey of psychological distress among Chinese people in the COVID-19 epidemic: implications and policy recommendations. Gen Psychiatr 2020; 33: e100213.

36. Zheng Z, Peng F, Xu B, et al. Risk factors of critical \& mortal COVID-19 cases: a systematic literature review and metaanalysis. J Infect 2020; 80: e16-e25. 
37. Schwartz T, Pines E. Coping with fatigue, fear, and panic during a crisis. Available at: https://hbr.org/2020/03/coping-withfatigue-fear-and-panic-during-acrisis (accessed 1 May 2020).

38. Bohlken J, Schömig F, Lemke M, et al. COVID-19 pandemic: stress experience of healthcare workers - a short current review. Psychiatr Prax 2020; 47: 190-197.

39. Kersten DJ, Makaryus AN. We may develop medications effective against COVID-19, but can we distribute them equitably? Health Equity 2020; 4: 290-291.

40. Farmer N, Wallen G, Baumer Y, et al. COVID-19: Growing health disparity gaps and opportunity for health behavior discovery? Health Equity 2020; 4: 316-319.

41. Lee AM, Wong JG, McAlonan GM, et al. Stress and psychological distress among SARS survivors 1 year after the outbreak. Can J Psychiatry 2007; 52: 233-240. 